\title{
Etude, caractérisation et optimisation de lampes flash microseconde et submicroseconde dans le domaine $200-300 \mathrm{~nm}$
}

\author{
J. M. Pouvesle et R. Viladrosa
}

GREMI, CNRS, Université d'Orléans, rue de Chartres, 45067 Orléans Cedex, France

\begin{abstract}
Résumé: Ce travail est consacré à l'optimisation, dans l'UV lointain, de lampes flash rapides (impulsions $\simeq 5 \mu \mathrm{s}$ ) traditionnelles . A dépôt d'énergie constant, le meilleur rendement dans le domaine 200-300 $\mathrm{nm}$ a été obtenu dans des mélanges $\mathrm{Xe}^{-\mathrm{O}_{2}}$ avec une augmentation de 1 'ordre de $50 \%$ par rapport aux résultats obtenus dans le xénon pur.

Abstract: This work reports the optimization, in the far $U V$, of fast (pulses $\simeq 5 \mu \mathrm{s}$ ) conventional flash lamps. At constant energy deposition, the best effeciency in the range 200-300 $\mathrm{nm}$ has been obtained in $\mathrm{Xe}-\mathrm{O}_{2}$ mixtures with an increase of the order of $50 \%$ compare with results obtained in pure xenon.
\end{abstract}

Le besoin de sources de forte puissance dans 1'UV se fait ressentir dans de nombreux domaines, tout particulièrement dans celui de la microélectronique, où il existe une forte demande liée aux différents traitements tels que recuit, dopage, photolithographie, dépôt ou polymérisation. L'emploi des lasers, principalement excimères, s'est vite généralisé, mais des développements récents $[1,2]$ ont montré que dans bien des cas, l'utilisation de sources incohérentes, en général moins onéreuses, pouvait parfaitement convenir pour peu qu'elles répondent à certaines spécifications spectrales et temporelles. Dans ce travail, mené en collaboration avec le CNET, nous avons étudié la possibilité d'optimiser des lampes flash classiques de manière à ce que le pourcentage de l'énergie rayonnée dans l'UV entre 200 et $300 \mathrm{~nm}$ soit maximum, avec des densités d'énergie sous flash de l'ordre du joule/cm ${ }^{2}$, des temps d'éclairs inférieurs à la microseconde et une surface rayonnante de plusieurs $\mathrm{cm}^{2}$.

Nous avons travaillé avec des flashes de 2 et $10 \mathrm{~cm}$ (diamètre 0.8 $\mathrm{cm}$ ), à déclenchement externe, directement reliés à un dispositif de mélange de gaz, avec lesquels nous avons pu étudier la réponse spectrale et en énergie de décharges créées dans des milieux très variés (mélanges à partir de : $\left.\mathrm{He}, \mathrm{Ar}, \mathrm{Kr}, \mathrm{Xe}, \mathrm{H} 2, \mathrm{~N}_{2}, \mathrm{O}_{2}, \mathrm{NO}\right)$ entre 1 et 1520 torrs, avec des pressions partielles d'additifs pouvant descendre jusqu'à qqu pour mille et des densités d'énergie déposée allant jusqu'à $11.25 \mathrm{~J} / \mathrm{cm}^{3}$. L'ensemble des résultats a pu être comparé à ceux obtenus dans des flashes Xénon, à une atmosphère, présentant des caractéristiques géométriques identiques.

Le meilleur rendement [3] dans l'UV lointain a été obtenu dans un mélange Xénon-0xygène (560 et 200 torr respectivement) (voir figure 1) avec environ $35 \%$ de 1 'énergie totale rayonnée dans le domaine $200-300 \mathrm{~nm}$, correspondant à une énergie déposée sous flash de 1 'ordre de $0.45 \mathrm{~J} / \mathrm{cm}^{2}$ 
dans ce même domaine de longueur d'onde. D'après le tableau $I$, on peut voir que cela représente une augmentation d'environ $50 \%$ par rapport à Xe pur dans les mêmes conditions de décharge.

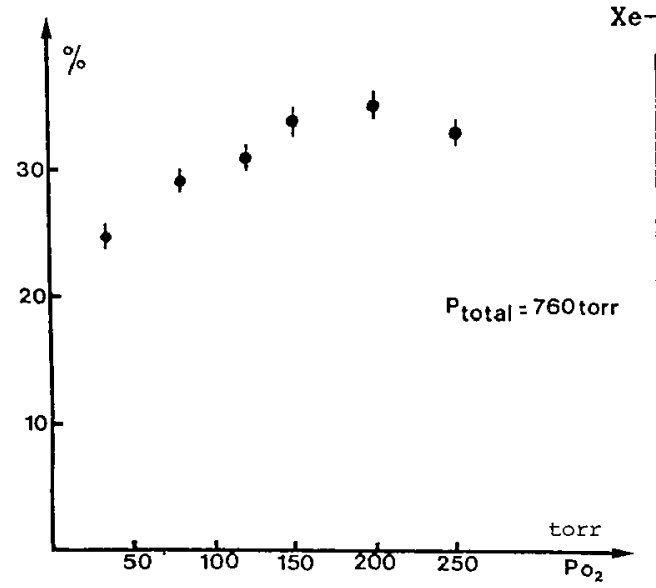

Tableau I: Comparaison du pourcentage de l'énergie globale rayonnée dans différents intervalles de longueurs d'onde dans Xe pur et dans un mélange $\mathrm{Xe}-02$ (560 - 200 torr)

Figure 1 : Pourcentage de I'énergie rayonnée dans l'intervalle 200-300 nm en fonction de la pression de $\mathrm{O}_{2}$ dans des mélanges $\mathrm{Xe}-\mathrm{O}_{2}$ à une pression totale de 760 torr.

\begin{tabular}{|c|c|c|}
\hline $\begin{array}{r}\lambda \\
n m\end{array}$ & $\begin{array}{c}\text { Xe pur } \\
\%\end{array}$ & $\mid \begin{array}{ccc}\mathrm{Xe} & + & \mathrm{O}_{2} \\
500 & & 200 \\
& 8 & \end{array}$ \\
\hline $200-305$ & 23.6 & 35.1 \\
\hline $305-370$ & 11.7 & 4.8 \\
\hline $370-550$ & 26.3 & 27.2 \\
\hline $550-695$ & 8.5 & 6.6 \\
\hline$>695$ & 29.9 & 26.3 \\
\hline
\end{tabular}

Dans un montage flash classique, la composition du mélange gazeux n'a que très peu d'influence sur la durée de l'impulsion lumineuse, fixée pratiquement uniquement par les paramètres externes du circuit de décharge ( 5 à $7 \mu \mathrm{s}$ dans nos conditions expérimentales). La réduction du temps d'éclair, à dépôt d'énergie constant, n'a été possible que moyennant la mise au point d'un dispositif de décharge rapide faisant appel à un système hybride ligne-capacité discrète de faible inductance travaillant à haute tension. A dépôt d'énergie et puissance rayonnée dans l'UV équivalents, nous avons pu obtenir des impulsions lumineuses allant jusqu'à $0.7 \mu \mathrm{s}$, les plasmas formés restants très homogènes. Il faut dans ce cas noter une ablation non négligeable des parois, avec apparition de raies de Si, diminuant la durée de vie du flash.

Cette étude a permis de clairement montrer la possibilité de développer des sources flash répondant aux spécifications mentionnées plus haut et correspondant aux besoins de la microélectronique.

[1] ESROM H. and KOGELSCHATZ U. Applied Surface Science 46 (1990) 158

[2] YOSHIZAWA K., TAKI M., TACHIBANA K., and MORIYAMA S. Appl. Phys. Lett. 59 (1991) 1678

[3] POUVESLE J.M., VILADROSA R.

Rapport Final, Contrat CNET n* 87 3B 0367909245

Ce travail a bénéficié du support de la convention CNET $\mathbf{n}^{*} 873 B \quad 036790$ 9245 